\title{
Prognostic factors of atrial fibrillation following elective coronary artery bypass grafting: the impact of quantified intraoperative myocardial ischemia
}

\author{
Efstratios N Koletsis ${ }^{1 \dagger}$, Christos Prokakis $^{1 * \dagger}$, James R Crockett $^{3+}$, Panagiotis Dedeilias ${ }^{2+}$, Matthew Panagiotou ${ }^{3 \dagger}$, \\ Nikolaos Panagopoulos ${ }^{1+}$, Nikolaos Anastasiou ${ }^{4+}$, Dimitrios Dougenis ${ }^{1+}$ and Efstratios Apostolakis ${ }^{5+}$
}

\begin{abstract}
Background: Atrial fibrillation (AF) occurs in 28-33\% of the patients undergoing coronary artery revascularization (CABG). This study focuses on both pre- and peri-operative factors that may affect the occurrence of AF. The aim is to identify those patients at higher risk to develop AF after CABG.

Patients and methods: Two patient cohorts undergoing CABG were retrospectively studied. The first group (group A) consisted of 157 patients presenting AF after elective CABG. The second group (group B) consisted of 191 patients without AF postoperatively.

Results: Preoperative factors presenting significant correlation with the incidence of post-operative AF included: 1) age $>65$ years $(p=0.029)$, 2) history of $\operatorname{AF}(p=0.022)$, 3) chronic obstructive pulmonary disease $(p=0.008)$, 4) left ventricular dysfunction with ejection fraction $<40 \%(p=0.015)$ and 5) proximal lesion of the right coronary artery $(p=0.023)$. The intraoperative factors that appeared to have significant correlation with the occurrence of postoperative AF were: 1) CPB-time $>120$ minutes $(p=0.011)$, 2) myocardial ischemia index $<0.27 \mathrm{ml} . \mathrm{m}^{2} / \mathrm{Kg} . \mathrm{min}$ $(p=0.011), 3)$ total positive fluid-balance during ICU-stay $(p<0.001), 4) \mathrm{FiO}_{2} / \mathrm{PO}_{2}>0,4$ after extubation and during the ICU-stay $(p=0.021)$, 5) inotropic support with doses $15-30 \mu \mathrm{g} / \mathrm{Kg} / \mathrm{min}(p=0.016), 6)$ long ICU-stay recovery for any reason $(p<0.001)$ and perioperative myocardial infarction $(p<0.001)$.

Conclusions: Our results suggest that the incidence of post-CABG atrial fibrillation can be predicted by specific preoperative and intraoperative measures. The intraoperative myocardial ischemia can be sufficiently quantified by the myocardial ischemia index. For those patients at risk we would suggest an early postoperative precautionary anti-arrhythmic treatment.
\end{abstract}

Keywords: post-CABG atrial fibrillation, cardiopulmonary bypass, coronary artery bypass grafting, CABG, Myocardial Ischemia Index, postoperative supraventricular arrhythmias, predictors

\section{Background}

Atrial Fibrillation (AF) remains the most common arrhythmia after cardiac surgery. Its incidence depends on patient's preoperative profile and the type of operation performed. AF occurs in approximately $28-33 \%$ of the patients undergoing coronary artery bypass grafting (CABG) [1-3] and in $30-63 \%$ of those operated for

\footnotetext{
*Correspondence: xristosprokakis@gmail.com

+ Contributed equally

'Cardiothoracic Surgery Department, University of Patras, School of Medicine, Patras, Greece

Full list of author information is available at the end of the article
}

coexisting ischemic heart and valve disease [3,4]. The majority of AF arrhythmias appear within the first 4-5 postoperative days and the peak frequency is in the $2^{\text {nd }}$ or $3^{\text {rd }}$ postoperative day $[5,6]$. It has been reported that patients with postoperative AF have longer Intensive Care Unit (ICU) stay, longer hospitalization, and higher incidence of re-admissions increasing the cost of hospitalization by $30 \%[3,7]$. This study is focused on the definition or pre- and peri-operative factors associated with the development of AF after CABG. The primary point is to find those patients at increased risk that may

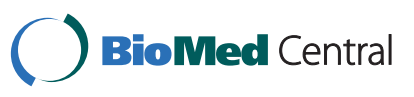

() 2011 Koletsis et al; licensee BioMed Central Ltd. This is an Open Access article distributed under the terms of the Creative Commons Attribution License (http://creativecommons.org/licenses/by/2.0), which permits unrestricted use, distribution, and reproduction in any medium, provided the original work is properly cited. 
benefit of a precautionary preoperative anti-arrhythmic treatment.

\section{Materials and methods Patients}

From 2002 to 2006514 patients were operated on for coronary artery disease at the $1^{\text {st }}$ Cardiac Surgery Department at "Evangelismos" General Hospital in Athens, and the Cardiothoracic Surgery Department at Patras University. One hundred and sixty six patients were excluded from further analysis because of the following exclusion criteria: 1) preoperative, chronic (duration $>3$ months) AF, 2) anti-arrhythmic treatment or history of cardiac arrhythmia other than AF, 3) concomitant heart valve disease other than trivial to mild ischemic mitral regurgitation $(1+$ or $2+/ 4+)$, 4) significant ischemic mitral regurgitation requiring mitral valve repair or substitution, 5) abnormal thyroid function or treatment for any thyroid disease, 6) acute or chronic renal failure (creatinine levels $\geq 200 \mathrm{mMol} / \mathrm{L}$ ), 7) symptomatic congestive heart failure or severe dysfunction of the left ventricle (EF $\leq$ $0.30), 8)$ administration of any other medication except those for coronary disease (b-blockers, nitrates, calcium channel blockers, and anti-platelets), 9) history of previous neurologic stroke or deficit, and 10) re-operation. The remaining 348 patients were divided in two groups. The first group (Group A) included 157 patients (45.12\%) undergoing CABG who developed postoperative AF within the first 10 postoperative days. The treatment of AF consisted of medical therapy and/or electrical cardioversion. The second group (Group B) included 191 patients (54.88\%) having the same operation but without the occurrence of postoperative AF. Methods and treatments were the same in both study groups. Oral antianginal medication was continued until the day of operation unless unstable angina was present. In this case continuous intravenous anti-anginal treatment was given until surgery.

\section{Surgical procedure}

All patients were operated on cardiopulmonary bypass. The distal anastomoses were performed first. The left internal mammary artery (LIMA) was exclusively used to bypass left anterior descending artery (LAD) stenoses whenever it was chosen as suitable (flow $>60 \mathrm{ml} / \mathrm{min}$ and sufficient length). Major saphenous vein grafts were used to bypass the diseased marginal (OM), diagonal (Diag) and/or right coronary artery (RCA). The proximal anastomoses were constructed during re-warming with the aorta de-clamped. Just after discontinuation of cardiopulmonary bypass and thereafter, in the ICU, a fluidbalance was daily recorded. During the ICU-stay and later on, in the ward, the ratio $\mathrm{FiO}_{2} / \mathrm{pO}_{2}$ was recorded to estimate the grade of hypoxemia. All patients were under surveillance in the ICU for the first 24-72 hours. Further observation for any arrhythmia development was carried out in the ward till discharge. When episodes of AF appeared, treatment consisted in amiodarone infusion with or without electrical cardioversion.

\section{Myocardial protection}

Myocardial protection was obtained using systemic hypothermia $\left(28^{\circ}-30^{\circ} \mathrm{C}\right)$ and intermittent administration of cold blood cardioplegia. Initial infusion of cardioplegia was $1000 \mathrm{ml}$ through the aortic root (antegrade). Thereafter it was infused via the coronary ostia and/or the grafts (after the completion of each distal anastomosis), in repeated doses of 300-400 $\mathrm{ml}$ at target intervals of 15-20 minutes. The pressure of cardioplegic perfusion was $100 \mathrm{mmHg}$, the temperature of cardioplegic solution was $6-8^{\circ}$ Celcius, and the infusion flow was $250 \mathrm{ml} / \mathrm{min}$. Therefore, the total volume of cardioplegia was mainly depended on the number of the distal anastomoses performed and generally on the length of aortic cross-clamp time. We estimated the myocardial injury related to myocardial protection by applying a mathematic model which included some factors known to present a strong relation with the development of AF: volume of cardioplegia, time between each cardioplegic delivery, temperature and body mass index. We called the final measure of this model the Myocardial Ischemia Index (MII) and it was estimated as follows:

MII $\infty\left[\mathrm{V}_{\mathrm{C}} \times \mathrm{F}_{\mathrm{C}} \times\left(\mathrm{P}_{\mathrm{D}}-\mathrm{P}_{\mathrm{S}}\right)\right] /\left[\right.$ B.M.I. $\times$ I.i. $\left.\times \mathrm{T}_{\mathrm{C}}\right]$, where:

1) $V_{C}=$ volume of cardioplegia)

2) $F_{C}=$ cardioplegia flow; fixed at $250 \mathrm{mls} / \mathrm{min}$. by protocol

3) $T_{C}=$ cardioplegia temperature; fixed at $6^{\circ} \mathrm{C}$ by protocol

4) $\left(\mathrm{P}_{\mathrm{D}}-\mathrm{P}_{\mathrm{S}}\right)=$ cardioplegia delivery pressure minus coronary sinus pressure; fixed by protocol at 100 $\mathrm{mmHg}$

5) I.i. = ischemia interval; time between each cardioplegia delivery for each anastomosis performed

6) B.M.I. = Body Mass Index; relative approximation to cardiac muscle mass.

Thus, considering that $\mathrm{F}_{\mathrm{C}},\left(\mathrm{P}_{\mathrm{D}}-\mathrm{P}_{\mathrm{S}}\right)$ and $\mathrm{T}_{\mathrm{C}}$ were constant and fixed by the protocol, this leaves us with the approximation:

MII $\infty \mathrm{V}_{\mathrm{C}} /($ B.M.I. $\times$ I.i.) with the units expressed in $\mathrm{mls} . \mathrm{m}^{2} / \mathrm{kg}$. min.

The MII was calculated, using this more abbreviated approximation, for each antegrade delivery and it was termed $\mathrm{MII}_{\text {ante }}$. For each patient both the minimum value $\left({ }_{\min } \mathrm{MII}_{\text {ante }}\right)$ and the average one $\left({ }_{\mathrm{av}} \mathrm{MII}_{\text {ante }}\right)$ resulting from the sum of the values for patient were calculated. 
Postoperative indices of myocardial infarction

The levels of serum myocardial enzymes (CK, CK-MB) were daily checked after surgery. Troponin I levels were not routinely checked. The diagnosis of myocardial infarction (MI) was based on the ECG alterations, the level of the enzymes and the results of cardiac echo. ORS widening persisting for more than 12 hours after surgery or new $\mathrm{Q}$ wave combined with positive enzyme values and echo evidence of new focal disturbances in myocardial performance pointed out the occurrence of perioperative MI.

\section{Statistical analysis}

All values are expressed as mean \pm standard deviation. Comparison of data among the two groups of patients was performed by the Pearson chi square test (asym 2-sided) and the Fischer exact test. Values less than 0.05 were considered statistically significant. All analyses were performed using the SPSS 16 statistical package.

\section{Results}

Tables 1 and 2 describe the patients' preoperative and main intra and post-operative characteristics respectively.

The incidence of postoperative atrial fibrillation for the total cohort of patients was $45.1 \%$ (157 out of 348 patients). Comparing the two groups of patients in relation to their preoperative characteristics we found that the parameters having statistically significant impact on the postoperative occurrence of AF were the following (table 3): 1) age $>65$ $(\mathrm{p}=0.029), 2)$ history of AF $(\mathrm{p}=0.022), 3)$ chronic obstructive pulmonary disease $(\mathrm{p}=0.008), 4)$ left ventricular dysfunction expressed by EF $<0.40(\mathrm{p}=0.015)$ and 5) proximal RCA stenosis ( $\mathrm{p}=0.023)$. The intra-, and postoperative parameters statistically related to the occurrence of postoperative AF were (table 4): 1) CPB-time above 120 minutes $(\mathrm{p}=0.011)$ (cross clamp time not statistically significant, $\mathrm{p}<0.05) 2$ ) ${ }_{\text {av }} \mathrm{MII}_{\text {ante }}$ value less than $0.27 \mathrm{ml}$. $\left.\mathrm{m}^{2} / \mathrm{Kg} \cdot \min (\mathrm{p}=0.011), 3\right)$ positive fluid balance during ICU recovery $(\mathrm{p}<0.001)$, 4) $\mathrm{FiO}_{2} / \mathrm{pO}_{2}$ ration $\leq 0.40$ during ICU stay $(\mathrm{p}=0.021), 5)$ high dose $(>15 \mu \mathrm{g} / \mathrm{Kg} / \mathrm{min})$ inotropic support $(\mathrm{p}=0.016)$, and ICU-stay $>48$ hour for any reason $(\mathrm{p}<0.001)$.

\section{Discussion}

AF is the result of the dispersion of atrial refractoriness resulting in multiple reentry wavelets in the atria [8]. In the postsurgical state of the heart several parameters may alter the refractoriness of adjacent atrial areas predisposing to reentry circuits and to the development of atrial fibrillation: inflammation [9], heightened sympathetic and vagal stimulation $[10,11]$, fluid overload and postoperative ventricular stunning resulting in atrial pressure elevation [12,13], chronic distention of the left atrium $[14,15]$, metabolic derangements such as hypoglycemia
Table 1 Patients' clinical and preclinical characteristics

\begin{tabular}{|c|c|c|}
\hline Clinical characteristics & Number of patients & Percentage \\
\hline \multicolumn{3}{|l|}{ Gender } \\
\hline Male & 297 & $85.30 \%$ \\
\hline Female & 51 & $14.70 \%$ \\
\hline \multicolumn{3}{|c|}{ Age: $62.2 \pm 9$ (43-82 years) } \\
\hline $41-55$ & 83 & $23.90 \%$ \\
\hline $56-65$ & 136 & $39.05 \%$ \\
\hline$\geq 65$ & 129 & $37.05 \%$ \\
\hline Diabetes & 49 & $14.10 \%$ \\
\hline History AF (<3 months) & 48 & $13.80 \%$ \\
\hline History MI & 131 & $37.70 \%$ \\
\hline Anterior Ml & 89 & $25.60 \%$ \\
\hline Posterior Ml & 42 & $12.10 \%$ \\
\hline COPD & 44 & $12.60 \%$ \\
\hline OPA & 48 & $13.80 \%$ \\
\hline Unstable angina & 30 & $8.60 \%$ \\
\hline Obesity (BMI > 30) & 43 & $12.40 \%$ \\
\hline Hypertension & 151 & $43.40 \%$ \\
\hline \multicolumn{3}{|l|}{ Preclinical characteristics } \\
\hline \multicolumn{3}{|l|}{ Diseased vessels } \\
\hline CAD-1 & 19 & $5.50 \%$ \\
\hline CAD-2 & 56 & $16.10 \%$ \\
\hline CAD-3 & 270 & $77.60 \%$ \\
\hline LMCAD & 34 & $9.80 \%$ \\
\hline \multicolumn{3}{|l|}{ E.F } \\
\hline $0.30-0.40$ & 53 & $15.20 \%$ \\
\hline $0.40-0.55$ & 64 & $18.40 \%$ \\
\hline$>0.55$ & 231 & $66.40 \%$ \\
\hline Mild MR & 22 & $6.30 \%$ \\
\hline L.A dilation $(>40 \mathrm{~mm}$ ) & 26 & $7.50 \%$ \\
\hline \multicolumn{3}{|l|}{ Proximal stenosis } \\
\hline Proximal LAD & 81 & $23.30 \%$ \\
\hline Proximal LCX & 114 & $32.80 \%$ \\
\hline Proximal RCA & 74 & $21.30 \%$ \\
\hline Dyslipidemia & 189 & $54.30 \%$ \\
\hline \multicolumn{3}{|l|}{ Medical treatment } \\
\hline Nitrates & 296 & $85.10 \%$ \\
\hline b-blockers & 258 & $74.10 \%$ \\
\hline $\mathrm{Ca}^{++}$blockers & 143 & $41.10 \%$ \\
\hline Anti-platelets & 284 & $81.60 \%$ \\
\hline
\end{tabular}

AF: atrial fibrillation, MI: myocardial infarction, COPD: chronic obstructive pulmonary disease, OPA: obstructive peripheral arteriopathy, BMI: body mass index, CAD: coronary artery disease, E.F: ejection fraction, MR: mitral regurgitation, L.A: left atrium, LAD: left anterior descending artery, LCX: circumflex artery, RCA: right coronary artery.

[16] and altered thyroid function, including both hyperand hypo-thyroidism [17], alterations of the cardiac structure and electrophysiological profile of the atria due to the surgical atrial trauma itself [5], and ischemic atrial injury $[18,19]$. 
Table 2 Patients' intra and postoperative characteristics

\begin{tabular}{|c|c|c|}
\hline Characteristic & Number of patients & Percentage \\
\hline \multicolumn{3}{|l|}{ CPB-time: $98 \pm 13 \min (43-158)$} \\
\hline CPB-time $<60$ & 35 & $10 \%$ \\
\hline CPB-time $60-120$ min & 237 & $68.10 \%$ \\
\hline CPB-time $>120 \mathrm{~min}$ & 76 & $21.90 \%$ \\
\hline \multicolumn{3}{|l|}{ Ischemia time: $47 \pm 16 \min (16-79)$} \\
\hline \multicolumn{3}{|c|}{ Myocardial Ischemia Index (M.I.I): $0.1-1.0 \mathrm{ml} . \mathrm{m}^{2} / \mathrm{Kg} . \mathrm{min}$} \\
\hline av.MII ante $\geq 0.5 \mathrm{ml} \cdot \mathrm{m}^{2} / \mathrm{Kg} \cdot \mathrm{min}$ & 104 & $29.90 \%$ \\
\hline av.MII ante $0.28-<0.49 \mathrm{ml} \cdot \mathrm{m}^{2} / \mathrm{Kg} \cdot \mathrm{min}$ & 176 & $50.60 \%$ \\
\hline av.MII ante $\leq 0.27 \mathrm{ml} \cdot \mathrm{m}^{2} / \mathrm{Kg} \cdot \mathrm{min}$ & 68 & $19.50 \%$ \\
\hline \multicolumn{3}{|l|}{ Bypasses performed } \\
\hline CABG-1 & 24 & $6.90 \%$ \\
\hline CABG-2 & 102 & $29.30 \%$ \\
\hline CABG-3 & 210 & $60.40 \%$ \\
\hline CABG-4 & 12 & $3.40 \%$ \\
\hline LIMA use & 312 & $89.70 \%$ \\
\hline Positive fluid balance & 207 & $59.50 \%$ \\
\hline Potassium Deficit & 276 & $79.30 \%$ \\
\hline \multicolumn{3}{|l|}{$\mathrm{FiO}_{2} / \mathrm{PO}_{2}$} \\
\hline$\leq 40$ & 302 & $86.80 \%$ \\
\hline$>40$ & 46 & $13.20 \%$ \\
\hline \multicolumn{3}{|l|}{ Inotropic support } \\
\hline Absent & 235 & $67.50 \%$ \\
\hline $3-15 \mu \mathrm{g} / \mathrm{kg} / \mathrm{min}$ & 62 & $17.80 \%$ \\
\hline$>15 \mu \mathrm{g} / \mathrm{kg} / \mathrm{min}$ & 51 & $14.70 \%$ \\
\hline Perioperative myocardial infarction & 19 & $5.50 \%$ \\
\hline \multicolumn{3}{|l|}{ ICU-recovery } \\
\hline$\leq 48$ hours & 279 & $80.20 \%$ \\
\hline$>48$ hours & 69 & $19.80 \%$ \\
\hline
\end{tabular}

CPB: Cardiopulmonary Bypass, M.I.I: Myocardial Ischemia Index, CABG: Coronary Artery Bypass Grafting, LIMA: left internal thoracic (mammary) artery, FiO ${ }_{2}$ : fraction of delivered $\mathrm{O}_{2}, \mathrm{PO}_{2}$ : arterial partial pressure of $\mathrm{O}_{2}$, ICU: Intensive Care Unit.

The intraoperative ischemia of the atrial wall has been considered as the most important factor related to the pathophysiological changes resulting in postoperative AF [20]. It has been shown that during a heart operation both the atrial septum and atrial wall remain warmer than the wall of the left ventricle $[4,21,22]$. Therefore, the protection of the atrial wall remains relatively inadequate compared to that of the left ventricular wall. Based on that consumption several trials have been carried out to identify the impact of different techniques of myocardial protection on the incidence of postoperative atrial arrhythmias without any clear benefit for any of the various strategies applied [23]. In our opinion the amount of cardioplegia is the most important factor related to the postoperative occurrence of AF. Jideus et al [24] showed that larger amounts of cardioplegia are related to lower incidence of postoperative AF. In our cohort of patients we observed a statistically significant relation between myocardial injury and postoperative AF. As shown in
Figure 1 describing the distribution of the av $\mathrm{MII}_{\text {ante }}$ values in relation to the frequency of postoperative AF, values of ${ }_{\text {av }} \mathrm{MII}_{\text {ante }}<0.27 \mathrm{mls} . \mathrm{m}^{2} / \mathrm{kg}$.min were related to a higher incidence of AF after CABG surgery ( $p=0.011$ ). Furthermore, when performing the same analysis using the lowest values of the $\mathrm{MII}_{\text {ante }}\left({ }_{\text {min }} \mathrm{MII}_{\text {ante }}\right)$ we observed that the ${ }_{\text {av }} \cdot \mathrm{MII}_{\mathrm{ante}}$ was a stronger predictor of postoperative atrial fibrillation than the ${ }_{\text {min }} \mathrm{MII}_{\text {ante }}$ indicating that one inadequate cardioplegia delivery is less important than more ones (Figure 1).

The prolonged CPB-time in cardiac surgery may result from any one or more of the following factors: delay in first placing the aortic cross clamp, prolonged cardioplegic deliveries, extended warm shot and prolonged reperfusion period, and not just prolonged ischemic intervals. In our study we found that CPB-time above 120 minutes was statistically related to postoperative AF. However, in contrast to other authors $[25,26]$ we haven't found any relation between the aortic cross clamp time and the 
Table 3 Impact of patients' preoperative characteristics on the development of post-CABG atrial fibrillation

\begin{tabular}{|c|c|c|c|}
\hline \multirow[t]{2}{*}{ Characteristic } & Group A (AF) & Group B (no AF) & Significance $(p)$ \\
\hline & 157 patients & 191 patients & \\
\hline Gender (male vs female) & & & $p=0.359$ \\
\hline Male & 137 & 160 & \\
\hline Female & 20 & 31 & \\
\hline \multicolumn{4}{|l|}{ Age } \\
\hline $41-55$ & 33 & 50 & NS \\
\hline $56-65$ & 56 & 80 & NS \\
\hline$>65$ & 68 & 61 & $p=0.029$ \\
\hline Diabetes & 25 & 24 & NS \\
\hline History AF & 29 & 19 & $p=0.022$ \\
\hline \multicolumn{4}{|l|}{ History MI } \\
\hline Anterior $\mathrm{Ml}$ & 41 & 48 & NS \\
\hline Posterior Ml & 18 & 24 & \\
\hline COPD & 28 & 16 & $p=0.008$ \\
\hline OPA & 19 & 29 & NS \\
\hline Unstable angina & 12 & 18 & NS \\
\hline Obesity (BMI > 30) & 24 & 19 & NS \\
\hline Hypertension & 72 & 79 & NS \\
\hline \multicolumn{4}{|l|}{ Diseased vessels } \\
\hline CAD-1 & 8 & 11 & NS \\
\hline CAD-2 & 26 & 30 & \\
\hline CAD-3 & 123 & 147 & \\
\hline$\underline{L M C A D}$ & 16 & 18 & \\
\hline \multicolumn{4}{|l|}{ E.F } \\
\hline $0.30-0.40$ & 32 & 21 & $p=0.015$ \\
\hline $0.40-0.55$ & 25 & 39 & NS \\
\hline$>0.55$ & 100 & 131 & NS \\
\hline Mild MR & 12 & 10 & NS \\
\hline L.A dilation (> $40 \mathrm{~mm}$ ) & 11 & 15 & \\
\hline \multicolumn{4}{|l|}{ Proximal stenosis } \\
\hline Proximal LAD & 43 & 38 & NS \\
\hline Proximal LCX & 55 & 59 & NS \\
\hline Proximal RCA & 42 & 32 & $p=0.023$ \\
\hline Dyslipidemia & 85 & 104 & NS \\
\hline \multicolumn{4}{|l|}{ Medical therapy } \\
\hline Nitrates & 137 & 159 & NS \\
\hline B-Blockers & 115 & 143 & \\
\hline $\mathrm{Ca}^{++}$- blockers & 68 & 75 & \\
\hline Anti-platelets & 131 & 153 & \\
\hline
\end{tabular}

AF: atrial fibrillation, MI: myocardial infarction, COPD: chronic obstructive pulmonary disease, OPA: obstructive peripheral arteriopathy, BMI: body mass index, CAD: coronary artery disease, E.F: ejection fraction, MR: mitral regurgitation, L.A: left atrium, LAD: left anterior descending artery, LCx: circumflex artery, RCA: right coronary artery. NS: not statistically significant $(p>0.05)$

frequency of postoperative AF. Furthermore, the quality of the coronary arteries and the number of bypasses performed, although reported as factors related to the length of ischemic time, showed no statistical influence on the outcome of postoperative AF.

Intraoperative infarction was statistically related to postoperative AF. This fact is also suggested by other authors
$[27,28]$. In our opinion it is possible that posterior infarcts are directly involved inducing ischemia of the atrial wall and septum while the anterior ones are indirectly implicated through the development of acute atrial enlargement. This last hypothesis is supported by the results of Knotzer et al [29] who observed that post-CABG high filling pressure in both atria due to ventricular stunning are 
Table 4 Impact of intra and postoperative parameters on the occurrence of post-CABG atrial fibrillation

\begin{tabular}{|c|c|c|c|}
\hline \multirow[t]{2}{*}{ Characteristic } & Group A (AF) & Group B (no AF) & Significance $(p)$ \\
\hline & 157 patients & 191 patients & \\
\hline \multicolumn{4}{|l|}{ CPB-time } \\
\hline$<60 \min$ & 12 & 23 & NS \\
\hline $60-120 \mathrm{~min}$ & 101 & 136 & NS \\
\hline$>120 \mathrm{~min}$ & 44 & 32 & $p=0.011$ \\
\hline \multicolumn{4}{|l|}{ M.I.I (ml.m²/Kg.min) } \\
\hline av.MII ante $\geq 0.5$ & 40 & 64 & NS \\
\hline av.MII ante $0.28-<0.49$ & 77 & 99 & NS \\
\hline av.MII ante $\leq 0.27$ & 40 & 28 & $p=0.011$ \\
\hline \multicolumn{4}{|l|}{$\overline{\text { CABG }}$} \\
\hline $1 \mathrm{graft}$ & 11 & 13 & NS \\
\hline 2 grafts & 46 & 56 & \\
\hline 3 grafts & 96 & 114 & \\
\hline 4 grafts & 4 & 8 & \\
\hline LIMA use & 145 & 167 & NS \\
\hline Positive fluid balance & 114 & 93 & $p<0.001$ \\
\hline Potassium deficit & 118 & 158 & NS \\
\hline \multicolumn{4}{|l|}{$\mathrm{FiO}_{2} / \mathrm{PO}_{2}$} \\
\hline$\leq 40$ & 129 & 173 & NS \\
\hline$>40$ & 28 & 18 & $p=0.021$ \\
\hline \multicolumn{4}{|l|}{ Inotropic support } \\
\hline No & 100 & 135 & NS \\
\hline $3-15 \mu \mathrm{g} / \mathrm{kg} / \mathrm{min}$ & 24 & 38 & NS \\
\hline$>15 \mu \mathrm{g} / \mathrm{kg} / \mathrm{min}$ & 33 & 18 & $p=0.016$ \\
\hline Perioperative MI & 16 & 3 & $p<0.001$ \\
\hline \multicolumn{4}{|l|}{ ICU-recovery } \\
\hline$\leq 48$ hours & 109 & 170 & NS \\
\hline$>48$ hours & 48 & 21 & $p<0.001$ \\
\hline
\end{tabular}

CPB: Cardiopulmonary Bypass, M.I.I: Myocardial Ischemia Index, CABG: Coronary Artery Bypass Grafting, LIMA: left internal thoracic (mammary) artery, FiO ${ }_{2}$ : fraction of delivered $\mathrm{O}_{2}, \mathrm{PO}_{2}$ : arterial partial pressure of $\mathrm{O}_{2}, \mathrm{MI}$ : Myocardial Infarction, ICU: Intensive Care Unit, NS: not statistically significant ( $p$ > 0.05)

statistically related to an increased incidence of postoperative AF. In the same study it has been shown that systemic hypoxia is also related to the development of postoperative AF. Such observation is also supported by our study. The systemic hypoxia may result from preexisting compromise of the patient's respiratory function with decreased pulmonary reserves or may be related to other parameters such as perioperative myocardial infarction causing interstitial pulmonary edema, or positive fluid balance. Positive fluid balance was found relative to the occurrence of postoperative AF in our study. A plausible explanation is that the positive fluid balance influences the development of AF through higher filling pressures of the left atrium and pulmonary congestion resulting in hypoxia. However, its role as a prognosticator is questionable. Both Osranek et al [15] and Place and colleagues [30] failed to identify net fluid balance either intra-operatively or postoperatively as a significant factor related to AF.

Postoperative low cardiac output has been reported as a parameter statistically related to postoperative AF [31].
In our opinion this observation is the result of the high inotropic support used in these patients to attain sufficient cardiac output. In this study indeed we found that high inotropic support (doses of Dopamine or Dobutamine, $>15 \mu \mathrm{g} / \mathrm{kg} / \mathrm{min}$ ) was statistically related to the incidence of postoperative AF.

A long ICU stay was found to be statistically related to the occurrence of AF after CABG. However this is a false presumption since a protracted ICU recovery may depend on other factors such us hypoxia, perioperative myocardial infarction and sepsis that predispose the patient to the development of postoperative arrhythmias.

We found that age $>65$ years was a significant predictor of AF after CABG. Advanced age has been documented as the most consistent predictor of AF after cardiac surgery $[1,2,15,27,28,31-33]$. Older patients present alterations in their atrial electrophysiological profile due to degenerative and inflammatory processes and therefore are more susceptible to the development of atrial fibrillation, especially in port cardiac surgery settings 


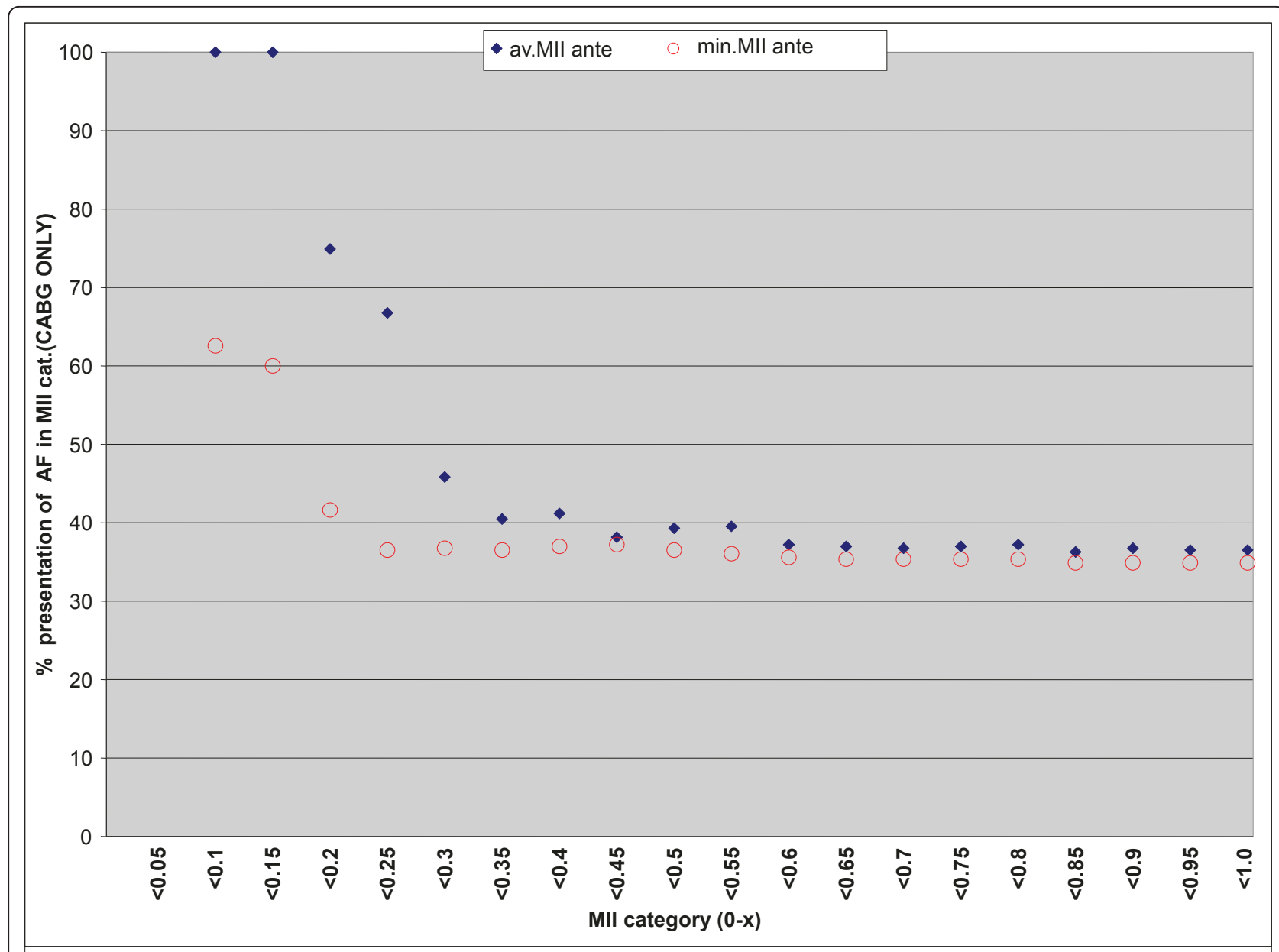

Figure 1 Distribution of the ${ }_{a v} \mathrm{MII}_{\text {ante }}$ and ${ }_{\min } \mathrm{MII}_{\text {ante }}$ values in relation to the frequency of postoperative AF. Note: avMIl ante: average value of Myocardial Ischemia Index, min MII ante: minimum value of Myocardial Ischemia Index.

[34]. This could also explain why patients with a history of episodes of AF prior to surgery have a greater risk to develop AF after surgery. In this study indeed all patients with episodes of AF within 3 months prior to surgery and $\mathrm{AF}$ after $\mathrm{CABG}$ belonged to the advanced age group (> 65 years old); on the contrary most patients with early preoperative onset AF and without post-CABG AF were less than 65 years old.

Both low ejection fraction and congestive heart failure prior to surgery have been recognized as independent predictors of AF $[2,4,35]$. These conditions result in chronic retention of blood in the atria, dilation of the atrial chambers and enlargement of their walls, providing an excellent substrate for the development of reentry circuits in the presence of intraoperative ischemia. This observation was also valid in our study, where an ejection fraction lower than $40 \%$ was statistically related to the incidence of AF after surgery.

Furthermore, we observed that patients presenting proximal lesions to the right coronary artery showed an increased incidence of AF which was statistically significant. Similar observations were made by Mendes et al [36] and Kolvekar and colleagues [19], supporting the role of diseased sino-atrial node and atrio-ventricular node arteries originating from the RCA in the development of AF.

Finally, patients suffering from COPD were at higher risk to develop AF. A plausible explanation is that patients suffering from impaired respiratory function are more likely to present hypoxia postoperatively especially if more contributing factors such as positive fluid balance, increased pulmonary artery and atrial pressures, perioperative myocardial infarction, lung atelectasis, infection and lung dysfunction related to the cardiopulmonary bypass, coexist.

\section{Conclusions}

Based on our results the incidence of postoperative atrial fibrillation can be predicted by specific preoperative and perioperative parameters. Advanced age 
represents an optimal substrate for the development of the arrhythmia especially when combined with increased stress of the atrial wall. This stress may result from chronic stress to the atrial wall such as the one observed in patients with low ejection fraction and congestive heart failure, intraoperative ischemic injury and postoperative stress factors like myocardial infarction and positive fluid balance. Intraoperative ischemic injury is sufficiently expressed by the M.I.I which is related to the magnitude of atrial mass (approximated here by the $\mathrm{BMI}$ ), the amount of cardioplegia delivered and the time between the cardioplegic deliveries. M.I.I represents an excellent predictor of postoperative AF after conventional coronary artery surgery. Patients presenting such predictors of AF may benefit from the precautionary early commencement of anti-arrhythmic treatment.

\section{Author details}

'Cardiothoracic Surgery Department, University of Patras, School of Medicine, Patras, Greece. ${ }^{2} 1^{\text {st }}$ Cardiac Surgery Department. "Evangelismos" General Hospital, Athens, Greece. ${ }^{3}$ Cardiac Surgery Department, Athens Medical Center, Greece. ${ }^{4}$ Department of Thoracic Surgery, $1^{\text {st }}$ IKA Hospital, Athens, Greece. ${ }^{5}$ Department of Cardiac Surgery, University of loannina, School of Medicine, loannina, Greece.

\section{Authors' contributions}

All authors: 1) have made substantial contributions to conception and design, or acquisition of data, or analysis and interpretation of data; 2) have been involved in drafting the manuscript or revising it critically for important intellectual content; and 3) have given final approval of the version to be published.

\section{Competing interests}

The authors declare that they have no competing interests.

Received: 24 May 2011 Accepted: 3 October 2011

Published: 3 October 2011

\section{References}

1. Zaman AG, Archbold RA, Helft G, Paul EA, Curzen NP, Mills PG: Atrial fibrillation after coronary artery bypass surgery: a model for preoperative risk stratification. Circulation 2000, 101:1403-1408.

2. Mathew JP, Fontes ML, Tudor IC, Ramsay J, Duke P, Mazer CD, Barash PG, Hsu PH, Mangano DT, Investigators of the Ischemia Research and Education Foundation; Multicenter Study of Perioperative Ischemia Research Group: A multicenter risk index for atrial fibrillation after cardiac surgery. JAMA 2004, 291:1720-1729.

3. Aranki SF, Shaw DP, Adams DH, Rizzo RJ, Couper GS, VanderVliet M, Collins JJ Jr, Cohn LH, Burstin HR: Predictors of atrial fibrillation after coronary artery surgery. Current trends and impact on hospital resources. Circulation 1996, 94:390-397.

4. Creswell LL, Schuessler RB, Rosenbloom M, Cox JL: Hazards of postoperative atrial arrhythmias. Ann Thorac Surg 1993, 56:539-549.

5. Maisel WH, Rawn JD, Stevenson WG: Atrial fibrillation after cardiac surgery. Ann Intern Med 2001, 135:1061-73.

6. Podrid PJ: Prevention of postoperative atrial fibrillation: what is the best approach? J Am Coll Cardiol 1999, 34:340-342

7. Kowey PR: Atrial arrhythmias after cardiac surgery: Sisyphus revisited? J Am Coll Cardiol 1999, 34:348-350.

8. Hogue CW Jr, Creswell LL, Gutterman DD, Fleisher LA, American College of Chest Physicians: Epidemiology, mechanisms and risks: American College of Chest Physicians guidelines for the prevention and management of postoperative atrial fibrillation after cardiac surgery. Chest 2005, 128(2 suppl):9S-16S
9. Ishii Y, Schuessler RB, Gaynor SL, Yamada K, Fu AS, Boineau JP, Damiano RJ $\mathrm{Jr}$ : Inflammation of atrium after cardiac surgery is associated with inhomogeneity of atrial conduction and atrial fibrillation. Circulation 2005, 111:2881-2888.

10. Liu L, Nattel S: Differing sympathetic and vagal effects on atrial fibrillation in dogs: role of refractoriness heterogeneity. Am J Physiol 1997, 273:H805-H816.

11. Hogue CW Jr, Domitrovich PP, Stein PK, Despotis GD, Re L, Schuessler RB, Kleiger RE, Rottman JN: PP interval dynamics before atrial fibrillation in patients after coronary artery bypass graft surgery. Circulation 1998, 98:429-434.

12. Kalus JS, Caron MF, White CM, Mather JF, Gallagher R, Boden WE, Kluger J: Impact of fluid balance on incidence of atrial fibrillation after cardiothoracic surgery. Am J Cardiol 2004, 94:1423-1425.

13. Sideris DA, Toumanidis ST, Tselepatiotis E, Kostopoulos K, Stringli T, Kitsiou T, Moulopoulos SD: Atrial pressure and experimental atrial fibrillation. Pacing Clin Elettrophysiol 1995, 18:1679-1685.

14. Everett TH, Li H, Mangrum JM, McRury ID, Mitchell MA, Redick JA Haines DE: Electrical, morphological and ultrastructural remodeling and reverse remodeling in a canine model of chronic atrial fibrillation. Circulation 2000, 102:1454-1460.

15. Osranek M, Fatema K, Qaddoura F, Al-Saileek A, Barnes ME, Bailey KR, Gersh BJ, Tsang TS, Zehr KJ, Seward JB: Left atrial volume predicts the risk of atrial fibrillation after cardiac surgery. J Am Coll Cardiol 2006, 48:779-786.

16. Vardas PE, Vemmos K, Sideris DA, Moulopoulos SD: Susceptibility of the right and left canine atria to fibrillation in hyperglycemia and hypoglycemia. J Electrocardiol 1993, 26:147-153.

17. Klemperer JD, Klein IL, Ojamaa K, Helm RE, Gomez M, Isom OW, Krieger KH: Triiodothyronine therapy lowers the incidence of atrial fibrillation after cardiac operations. Ann Thorac Surg 1996, 61:1323-1327.

18. Smith PK, Buhrman WC, Levett JM, Ferguson TB Jr, Holman WL, Cox JL: Supreventricular conduction abnormalities following cardiac operations: a complication of inadequate atrial preservation. J Thorac Cardiovasc Surg 1983, 85:105-115.

19. Kolvekar S, D'Souza A, Akhtar P, Reek C, Garratt C, Spyt T: Role of atrial ischemia in development of atrial fibrillation following coronary artery bypass surgery. Eur J Cardiothorac Surg 1997, 11:70-75.

20. Carmelo M, Smith P: Critical Care for the Adult Cardiac Patient. In Sabiston and Spencer Surgery of the Chest.. 7 edition. Edited by: Sellke F, del Nido P, Swanson S. Philadelphia, Elsevier/Saunders; 2005:1043-1045.

21. Chen $X Z$, Newman $M$, Rosenfeldt FL: Internal cardiac cooling improves atrial preservation: electrophysiological and biochemical assessment. Ann Thorac Surg 1988, 46:406-411.

22. Mullen JC, Khan N, Weisel RD, Christakis GT, Teoh KH, Madonik M, Mickle DA, Ivanov J: Atrial activity during cardioplegia and postoperative arrhythmias. J Thorac Cardiovasc Surg 1987, 94:558-565.

23. Creswell LL, Alexander JC Jr, Ferguson TB Jr, Lisbon A, Fleisher LA, American College of Chest Physicians: Intraoperative interventions: American College of Chest Physicians guidelines for the prevention and management of postoperative atrial fibrillation after cardiac surgery. Chest 2005, 128(2 suppl):28S-35S.

24. Jidéus L, Blomström P, Nilsson L, Stridsberg M, Hansell P, BlomströmLundqvist C: Tachyarrhythmias and triggering factors for atrial fibrillation after coronary artery by pass operation. Ann Thorac Surg 2000, 69:1064-1069.

25. Mathew JP, Parks R, Savino JS, Friedman AS, Koch C, Mangano DT, Browner WS, et al: Atrial fibrillation following coronary artery bypass graft surgery: predictors, outcomes and resource utilization. Multicenter Study of Perioperative Ischemia Research Group. JAMA 1996, 276:300-306.

26. Caretta Q, Mercanti CA, De Nardo D, Chiarotti F, Scibilia G, Reale A Marino B: Ventricular contraction defects and atrial fibrillation after coronary artery bypass grafting. Multivariate analysis of preoperative, intraoperative and postoperative variables. Eur Heart J 1991, 12:1107-1111.

27. Almassi GH, Schowalter $T$, Nicolosi AC, Aggarwal A, Moritz TE, Henderson WG, Tarazi R, Shroyer AL, Sethi GK, Grover FL, Hammermeister KE: Atrial fibrillation after cardiac surgery. A major morbid event? Ann Surg 1997, 226:501-511.

28. Nakai T, Lee RJ, Schiller NB, Bellows WH, Dzankic S, Reeves J, Romson J, Ferguson S, Leung JM: The relative importance of left atrial function 
versus dimension in predicting atrial fibrillation after coronary artery bypass graft surgery. Am Heart J 2002, 143:181-186.

29. Knotzer H, Mayr A, Ulmer H, Lederer W, Schobersberger W, Mutz N, Hasibeder W: Tachyarrhythmias in a surgical intesive care unit: a case controlled epidemiologic study. Intesive Care Med 2000, 26:908-914.

30. Place DG, Peragallo RA, Carroll J, Cusimano RJ, Cheng DC: Postoperative atrial fibrillation: A comparison of off pump coronary artery bypass surgery and conventional coronary artery bypass graft surgery. J Cardiothorac Vasc Anesth 2002, 16:144-148.

31. Amar D, Shi W, Hogue CW Jr, Zhang H, Passman RS, Thomas B, Bach PB, Damiano R, Thaler HT: Clinical prediction rule for atrial fibrillation after coronary artery bypass grafting. J Am Coll Cardiol 2004, 44:1248-1253.

32. Villareal RP, Hariharan R, Liu BC, Kar B, Lee W, Elayda M, Lopez JA, Rasekh A, Wilson JM, Massumi A: Postoperative atrial fibrillation and mortality after coronary artery bypass surgery. J Am Coll Cardiol 2004, 43:742-748.

33. Auer J, Weber T, Berent R, Ng CK, Lamm G, Eber B: Risk factors of postoperative atrial fibrillation after cardiac surgery. J Card Surg 2005, 20:425-431.

34. Allessie MA, Boyden PA, Camm AJ, Kléber AG, Lab MJ, Legato MJ, Rosen MR, Schwartz PJ, Spooner PM, Van Wagoner DR, Waldo AL: Pathophysiology and prevention of atrial fibrillation. Circulation 2001, 103:769-777.

35. Mayr A, Knotzer H, Pajk W, Luckner G, Ritsch N, Dünser M, Ulmer H, Schobersberger W, Hasibeder W: Risk factors associated with new onset tachyarrhytmias after cardiac surgery - a retrospective analysis. Acta Anaesthesiol Scand 2001, 45:543-549.

36. Mendes LA, Connelly GP, McKenney PA, Podrid PJ, Cupples LA, Shemin RJ, Ryan TJ, Davidoff R: Right coronary artery stenosis: an independent predictor of atrial fibrillation after coronary artery bypass surgery. J Am Coll Cardiol 1995, 25:198-202.

doi:10.1186/1749-8090-6-127

Cite this article as: Koletsis et al.: Prognostic factors of atrial fibrillation following elective coronary artery bypass grafting: the impact of quantified intraoperative myocardial ischemia. Journal of Cardiothoracic Surgery 2011 6:127.

\section{Submit your next manuscript to BioMed Central and take full advantage of:}

- Convenient online submission

- Thorough peer review

- No space constraints or color figure charges

- Immediate publication on acceptance

- Inclusion in PubMed, CAS, Scopus and Google Scholar

- Research which is freely available for redistribution

Submit your manuscript at www.biomedcentral.com/submit 